\title{
Monographs, a Category of Graph Structures
}

\author{
Thierry Boy de la Tour \\ CNRS and University Grenoble Alpes, LIG Lab. Grenoble, France, \\ thierry.boy-de-la-tour@imag.fr
}

\begin{abstract}
Does a graph necessarily have nodes? May an edge be adjacent to itself and be a self-loop? These questions arise in the study of graph structures, i.e., monadic many-sorted signatures and the corresponding algebras. A simple notion of monograph is proposed that generalizes the standard notion of directed graph and can be drawn consistently with them. It is shown that monadic many-sorted signatures can be represented by monographs, and that the corresponding algebras are isomorphic to the monographs typed by the corresponding signature monograph. Monographs therefore provide a simple unifying framework for working with monadic algebras. Their simplicity is illustrated by deducing some of their categorial properties from those of sets.
\end{abstract}

Keywords: Universal algebra · Monadic signatures · Category theory

\section{Introduction}

Many different notions of graphs are used in mathematics and computer science: simple graphs, directed graphs, multigraphs, hypergraphs, etc. One favourite notion in the context of logic and rewriting is that also known as quivers, i.e., structures of the form $(N, E, s, t)$ where $N, E$ are sets and $s, t$ are functions from $E$ (edges) to $N$ (nodes), identifying the source and target tips of every edge (or arrow). One reason for this is that the category of quivers is isomorphic to the category of $\Sigma_{\mathrm{g}}$-algebras, where $\Sigma_{\mathrm{g}}$ is the signature with two sorts nodes and edges and two operator names src and tgt of type edges $\rightarrow$ nodes. In conformity with this tradition, by graph we mean quiver throughout this paper.

In order to conveniently represent elaborate data structures it is often necessary to enrich the structure of graphs with other objects: nodes or edges may be labelled with elements from a fixed set, or with the elements of some algebra, or graphs may be typed by another graph (i.e., a graph comes with a morphism from itself to this other graph, considered as its type). An interesting example can be found in [4] with the notion of E-graphs, since some of these new objects are also considered as edges or nodes. More precisely, an E-graph is an algebra whose signature $\Sigma_{\mathrm{e}}$ can be represented by the following graph:

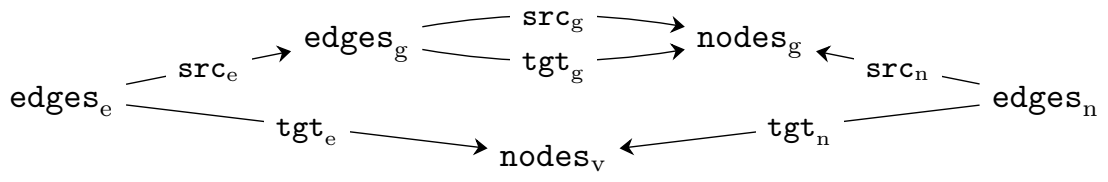


The names given to the sorts and operators help to understand the structure of the $\Sigma_{\mathrm{e}}$-algebras: the edges $\mathrm{g}_{\mathrm{g}}$ relate the nodes $\mathrm{g}$ among themselves, the edges $\mathrm{n}_{\mathrm{n}}$ relate the nodes $\mathrm{g}_{\mathrm{g}}$ to the nodes $\mathrm{s}_{\mathrm{v}}$, and the edges $\mathrm{e}_{\mathrm{e}}$ relate the edges $\mathrm{s}_{\mathrm{g}}$ to the nodes $\mathrm{s}_{\mathrm{v}}$. These extra edges allow values (elements of nodes ${ }_{\mathrm{v}}$ ) to be attached to edges and nodes of the inner graph. But then we see that in E-graphs some edges can be adjacent to other edges. This is non standard, but we may still accept such structures as some form of graph, if only because we understand how they can be drawn.

Hence the way of generalizing the notion of graphs seems to involve a generalization of the signature of graphs considered as algebras. This path has been followed by Michael Löwe in [7, where a graph structure is defined as a monadic many-sorted signature. Indeed in the examples above, and in many examples provided in [7, all operators have arity 1 and can therefore be considered as edges from their domain to their range sort. Is this the reason why they are called graph structures? But the example above shows that, if $\Sigma_{\mathrm{e}}$-algebras are interpreted as graphs of some form, these are very different from the graph $\Sigma_{\mathrm{e}}$. Besides, it is not convenient that our understanding of such structures should be based on syntax, i.e., on the particular names given to objects in the signature.

Furthermore, it is difficult to see how the algebras of some very simple monadic signatures can be interpreted as graphs of any form. Take for instance $\Sigma_{\mathrm{g}}$ and reverse the target function to tgt : nodes $\rightarrow$ edges. Then there is a symmetry between the sorts nodes and edges, which means that in an algebra of this signature nodes and edges would be objects of the same nature. Is this still a graph? Can we draw it? Worse still, if the two sorts are collapsed into one, does it mean that a node/edge can be adjacent to itself?

We may address these problems by restricting graph structures to some class of monadic signatures whose algebras are guaranteed to behave in an orthodox way, say by exhibiting clearly separated edges and nodes. But this could be prone to arbitrariness, and it would still present another drawback: that the notion of graph structure does not easily give rise to a category. Indeed, it is difficult to define morphisms between algebras of different signatures, if only because they can have any number of carrier sets.

The approach adopted here is rather to reject any structural distinction between nodes and edges, to gather them all in a single carrier set and to rely on a unique function to distinguish them. For this reason, the resulting structures are called monographs. The definitions of monographs and their morphisms, given in Section 3, are thus quite simple although for reasons that will only be made clear in Section 5 we use sequences of ordinal length, defined in Section 2

Prior to examining their mathematical properties, we show in Section 4 that these structures deserve to be considered as "graphs" since they can be represented by drawings, provided of course that they are finite (in a strong sense). In particular, such drawings correspond to the standard way of drawing a graph, for those monographs that can be identified with standard graphs.

The relationship between monographs and graph structures (monadic signatures) is explored in Section 5. using relevant notions from category theory 
defined in Section 2. This gives rise to an isomorphism-dense embedding of monographs into many-sorted monadic signatures, that will expose a fundamental difference between them. This result is used in Section 6 to exhibit isomorphisms between the categories of (partitioned) algebras of all graph structures and all slice categories of monographs, i.e., the categories of typed monographs.

As a result of their simplicity the category of monographs and some of its subcategories can easily be shown to share a number of properties with Graphs, as illustrated in Section 7, Concluding remarks are given in Section 8 The missing proofs can be found in $[3]$.

\section{Notations and Definitions}

For any sets $A, B$, any relation $r \subseteq A \times B$ and any subset $X \subseteq A$, we write $r[X]$ for the set $\{y \in B \mid x \in X \wedge(x, y) \in r\}$. For any $x \in A$, by abuse of notation we write $r[x]$ for $r[\{x\}]$. We write $r^{-1}$ for the relation $\{(y, x) \mid(x, y) \in r\} \subseteq B \times A$. If $r$ is functional we write $r(x)$ for the unique element of $r[x]$, and if $q \subseteq B^{\prime} \times C$ is also functional for sets $B^{\prime}$ and $C$ such that $r[A] \subseteq B^{\prime}$, we write $q \circ r$ for the functional relation $\{(x, q(r(x))) \in A \times C \mid x \in A\}$.

A function $f: A \rightarrow B$ is a triple $(A, r, B)$ where $A$ and $B$ are sets, respectively called the domain and codomain of $f$, and $r \subseteq A \times B$ is a functional relation. Hence $A$ must be the set $\{x \mid(x, y) \in r\}$ and $B$ may be any superset of the image $r[A]$, also denoted $f[A]$ (and generally $f[X]$ and $f(x)$ stand for $r[X]$ and $r(x)$ respectively). If $A=\varnothing$ and the codomain $B$ can be determined from the context then we write $\varepsilon$ for $f=(\varnothing, \varnothing, B)$. If $g=(B, q, C)$ is a function, then $g \circ f \stackrel{\text { def }}{=}(A, q \circ r, C)$. We may also compose a functional relation with a function, that then denotes its underlying functional relation, so that $q \circ f=g \circ r=q \circ r$.

More generally, any object and its obvious underlying object will be written similarly, i.e., the forgetful functor will be omitted, whenever the ambiguity can easily be lifted from the context. Category theoretic concepts and notations will be consistent with [1, unless stated otherwise. In particular, $\mathrm{id}_{A}$ denotes the identity morphism of the object $A$ in any category, except in Sets where it is denoted $\operatorname{Id}_{A}$ (the identity function of $A$ ) as a way of reminding the reader that $A$ is a set. In Sets the standard product $\times$, projections $\pi_{1}$ and $\pi_{2}$ and coproduct + are used. For functional relations $f, g$ with the same domain $A$, let $\langle f, g\rangle(x) \stackrel{\text { def }}{=}(f(x), g(x))$ for all $x \in A$; if $f: A \rightarrow B$ and $g: A \rightarrow C$ are functions then $\langle f, g\rangle: A \rightarrow B \times C$ is the unique function such that $\pi_{1} \circ\langle f, g\rangle=f$ and $\pi_{2} \circ\langle f, g\rangle=g$.

Isomorphism between objects in a category, or between categories, is denoted by the symbol $\simeq$. For any two categories $\boldsymbol{A}$ and $\boldsymbol{B}$, a functor $F: \boldsymbol{A} \rightarrow \boldsymbol{B}$ is faithful (resp. full) if $F$ is injective (resp. surjective) from the set of $\boldsymbol{A}$-morphisms from $X$ to $Y$ to the set of $\boldsymbol{B}$-morphisms from $F X$ to $F Y$, for all $\boldsymbol{A}$-objects $X$ and $Y$. If $F$ is faithful and injective on objects, then it is an embedding. $F$ is isomorphism-dense if for every $\boldsymbol{B}$-object $Y$ there exists an $\boldsymbol{A}$-object $X$ such that $F X \simeq Y$. Categories $\boldsymbol{A}$ and $\boldsymbol{B}$ are equivalent, written $\boldsymbol{A} \cong \boldsymbol{B}$, if there is a full, faithful and isomorphism-dense functor from one to the other. 
For any object $T$ of $\boldsymbol{A}$, the slice category ${ }^{1} \boldsymbol{A} / T$ has as objects the morphisms of codomain $T$ of $\boldsymbol{A}$, as morphisms from object $f: A \rightarrow T$ to object $g: B \rightarrow T$ the morphisms $k: A \rightarrow B$ of $\boldsymbol{A}$ such that $g \circ k=f$, and the composition of morphisms in $\boldsymbol{A} / T$ is defined as the composition of the underlying morphisms in $\boldsymbol{A}$. $T$ is a terminal object of $\boldsymbol{A}$ if for every object $A$ of $\boldsymbol{A}$ there is a unique $f: A \rightarrow T$. It is easy to see that $\operatorname{id}_{T}$ is a terminal object of $\boldsymbol{A} / T$, and that if $T$ is a terminal object of $\boldsymbol{A}$ then $\boldsymbol{A} \simeq \boldsymbol{A} / T$.

Since edges will have arbitrary lengths, including infinite ones, we use the notion of ordinal and refer to [1] for their properties. An ordinal is a set $\alpha$ such that every element of $\alpha$ is a subset of $\alpha$, and such that the restriction of the membership relation $\in$ to $\alpha$ is a strict well-ordering of $\alpha$ (every non empty subset of $\alpha$ has a minimal element). Every member of an ordinal is an ordinal, and we write $\lambda<\alpha$ for $\lambda \in \alpha$. For any two ordinals $\alpha, \beta$ we have either $\alpha<\beta$, $\alpha=\beta$ or $\alpha>\beta$. Every ordinal $\alpha$ has a successor $\alpha \cup\{\alpha\}$, denoted $\alpha+1$. Natural numbers $n$ are identified with finite ordinals, so that $n=\{0,1, \ldots, n-1\}$ and $\omega=\{0,1, \ldots\}$ is the smallest infinite ordinal.

Definition 2.1 ( $E$-sequences $s$ of length $\lambda$, elements $s_{\iota}, x \mid s$ ). For any set $E$ and ordinal $\lambda$, an $E$-sequence $s$ of length $\lambda i s$ an element of $E^{\lambda}$, i.e., $a$ function $s: \lambda \rightarrow E$. For any $s \in E^{\lambda}$ and $\iota<\lambda$, the image $s(\iota)$ is written $s_{\iota}$. If $\lambda$ is finite and non zero then $s$ can be described as $s=s_{0} \cdots s_{\lambda-1}$. For any $x \in E$ we write $x \mid s$ and say that $x$ occurs in $s$ if there exists $\iota<\lambda$ such that $s_{\iota}=x$.

Take for instance $E=\{x, y\}$, then $s=x y x$ describes the $E$-sequence of length $3=\{0,1,2\}$ such that $s_{0}=s_{2}=x$ and $s_{1}=y$. We have $x \mid x y x$ and $y \mid x y x$.

Note that there is no set of all $E$-sequences, hence the following notions.

Definition 2.2 (sets $E^{<\alpha}$, functions $f^{<\alpha}$ ). For any set $E$ and ordinal $\alpha$, let

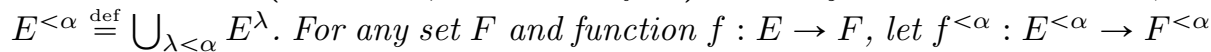
be the function defined by $f^{<\alpha}(s) \stackrel{\text { def }}{=} f \circ s$ for all $s \in E^{<\alpha}$.

Thus $s=x y x$ is an element of $E^{<4}, E^{<5} \ldots$ but not of $E^{<3}=E^{0} \cup E^{1} \cup E^{2}$. If $f: E \rightarrow E$ is the function that swaps $x$ and $y(f=(x y)$ in cycle notation), then $f^{<4}(s)_{0}=(f \circ s)_{0}=f\left(s_{0}\right)=y$, etc., hence $f^{<4}(s)=y x y$.

We have $E^{<0}=\varnothing$ and $E^{<1}=E^{0}=\{\varepsilon\}$, i.e., the function $\varepsilon$ (with codomain $E$ ) is the only $E$-sequence of length 0 . It is obvious that for any $s \in E^{<\alpha}$ and any ordinal $\beta \geqslant \alpha$, we have $s \in E^{<\beta}$ and $f^{<\beta}(s)=f^{<\alpha}(s)$. If $f: E \rightarrow F$ and $g: F \rightarrow G$ then $(g \circ f)^{<\alpha}=g^{<\alpha} \circ f^{<\alpha}$.

If $s$ and $s^{\prime}$ are respectively $E$ - and $F$-sequences of length $\lambda$, then they are both functions with domain $\lambda$ hence there is a function $\left\langle s, s^{\prime}\right\rangle$ of domain $\lambda$. Thus $\left\langle s, s^{\prime}\right\rangle$ is an $(E \times F)$-sequence of length $\lambda$, and then $\pi_{1}^{<\alpha}\left(\left\langle s, s^{\prime}\right\rangle\right)=\pi_{1} \circ\left\langle s, s^{\prime}\right\rangle=s$ and similarly $\pi_{2}^{<\alpha}\left(\left\langle s, s^{\prime}\right\rangle\right)=s^{\prime}$ for all $\alpha>\lambda$.

If $f: E \rightarrow F$ and $g: E \rightarrow G$ then $\langle f, g\rangle: E \rightarrow F \times G$, hence for all $s \in E^{<\alpha}$ of length $\lambda<\alpha$ we have $\langle f, g\rangle^{<\alpha}(s)=\langle f, g\rangle \circ s=\langle f \circ s, g \circ s\rangle=\left\langle f^{<\alpha}(s), g^{<\alpha}(s)\right\rangle$ is an $(F \times G)$-sequence of length $\lambda$.

\footnotetext{
${ }^{1}$ This is called the comma category of $\boldsymbol{A}$ over $T$ in [5, Definition 4.19], and the category of objects over $T$ in [1, Exercise $3 \mathrm{~K}]$. We adopt the terminology and notation of [4].
} 


\section{Categories of Monographs}

We may now define the structure of monographs and some related notions. These definitions will be illustrated by a running example and will be followed by a number of basic facts on monographs and a comparison with graphs.

In graphs, edges are arbitrary objects to which are associated two arbitrary objects called nodes. We may thus associate to each edge a sequence of nodes of length 2. In monographs, the corresponding sequences may contain arbitrary edges and have arbitrary lengths.

Definition 3.1 (monographs, grade, edges $\mathrm{E}_{a}$, length $\ell_{a}(x)$, trace $\left.\operatorname{tr}(A)\right)$. For any ordinal $\alpha$, an $\alpha$-monograph $A$ is a pair $(E, a)$ where $E$ is a set whose elements are called edges of $A$, and $a \subseteq E \times E^{<\alpha}$ is a functional relation, called the map of $A$. A pair $A=(E, a)$ is a monograph if it is an $\alpha$-monograph for some ordinal $\alpha$; we then say that $\alpha$ is an ordinal for $A$. The grade of $A$ is the smallest ordinal for $A$. Monographs will usually be denoted by upper-case letters $(A, B, \ldots)$, their map by the corresponding lower-case letter $(a, b, \ldots)$ and their set of edges $\mathrm{E}_{a}, \mathrm{E}_{b}, \ldots$

The length $\ell_{a}(x)$ of an edge $x \in \mathrm{E}_{a}$ is the length of $a(x)$, i.e., the unique ordinal $\lambda$ such that $a(x) \in \mathrm{E}_{a}^{\lambda}$. The trace of $A$ is the set $\operatorname{tr}(A) \stackrel{\text { def }}{=} \ell_{a}\left[\mathrm{E}_{a}\right]$. For any set $O$ of ordinals, an $O$-monograph ${ }^{2} A$ is a monograph such that $\operatorname{tr}(A) \subseteq O$.

Take for instance the monograph $A=(\{x, y\}, a)$ where $a=\{(x, x y x),(y, y x y)\}$ (i.e., $a(x)=x y x$ and $a(y)=y x y$ ), then $A$ is a 4-monograph and more precisely a $\{3\}$-monograph since $\operatorname{tr}(A)=\{3\}$, but $A$ is not a 3 -monograph, hence the grade of $A$ is 4 .

A monograph $A$ is essentially defined by its map $a$, since $\mathrm{E}_{a}=\{x \mid(x, y) \in a\}$. But $a$ is only a functional relation and not a function; there is no codomain to artificially separate monographs that have the same map. This means in particular that any $\alpha$-monograph is a $\beta$-monograph for all $\beta \geqslant \alpha$. It is easy to see that there always exists an ordinal for any two monographs, and indeed for any set of monographs (e.g. the sum of their grades).

Definition 3.2 (adjacency, self-loops, nodes $\mathrm{N}_{a}$, standard monographs). For any monograph $A$ and edges $x, y \in \mathrm{E}_{a}, x$ is adjacent to $y$ if $y \mid a(x)$. A self-loop is an edge $x$ that is adjacent only to $x$, i.e., such that $a(x)$ is an $\{x\}$ sequence. $A$ node is an edge of length 0 , and the set of nodes of $A$ is written $\mathrm{N}_{a}$. $A$ is standard if $a(x)$ is a $\mathrm{N}_{a}$-sequence for all $x \in \mathrm{E}_{a}$.

We see on the running example that $a(x)=x y x$, hence $x$ is adjacent to $y$ and to itself, but is not a self-loop. Similarly, $a(y)=y x y$ yields that $y$ is adjacent to $x$ and to itself, but is not a self-loop. $A$ has two edges but no nodes, hence $A$ is not standard.

A monograph $A$ is not normally defined by its adjacency relation $y \mid a(x)$ on edges, since the sequences $a(x)$ may not be uniquely determined by this relation.

\footnotetext{
${ }^{2}$ Note that any ordinal $\alpha$ is a set of ordinals, and that a monograph $A$ is an $\alpha$ monograph iff $\operatorname{tr}(A) \subseteq \alpha$.
} 
The adjacency relation may not be symmetric: a node is never adjacent to any edge, while edges may be adjacent to nodes.

Definition 3.3 (morphisms of monographs). A morphism $f$ from monograph $A=\left(\mathrm{E}_{a}, a\right)$ to monograph $B=\left(\mathrm{E}_{b}, b\right)$, denoted $f: A \rightarrow B$, is a function $f: \mathrm{E}_{a} \rightarrow \mathrm{E}_{b}$ such that $f^{<\alpha} \circ a=b \circ f$, where $\alpha$ is any ${ }^{3}$ ordinal for $A$.

Building on the running example, we consider the function $f=(x y)$ from $\mathrm{E}_{a}$ to $\mathrm{E}_{a}$, we see that $f^{<4} \circ a(x)=f^{<4}(x y x)=y x y=a(y)=a \circ f(x)$ and similarly that $f^{<4} \circ a(y)=f^{<4}(y x y)=x y x=a(x)=a \circ f(y)$, hence $f^{<4} \circ a=a \circ f$ and $f$ is therefore a morphism from $A$ to $A$. Since $f \circ f=\operatorname{Id}_{\mathrm{E}_{a}}$ is obviously the identity morphism of $A$ then $f$ is an isomorphism.

The length of edges are preserved by morphisms: if $f$ is a morphism from $A$ to $B$ then for all $x \in \mathrm{E}_{a}, \ell_{b}(f(x))$ is the length of the $\mathrm{E}_{b}$-sequence $b \circ f(x)=f^{<\alpha_{\circ}}$ $a(x)$, whose length is the same as the $\mathrm{E}_{a}$-sequence $a(x)$, i.e., $\ell_{a}(x)=\ell_{b}(f(x))$. Hence $\operatorname{tr}(A) \subseteq \operatorname{tr}(B)$, and the equality holds if $f$ is surjective. This means that if $B$ is an $O$-monograph then so is $A$. This also means that the grade of $B$ is at least that of $A$, hence that every ordinal for $B$ is an ordinal for $A$.

Given morphisms $f$ from $A$ to $B$ and $g$ from $B$ to $C$, we see that $g \circ f$ is a morphism from $A$ to $C$ by letting $\alpha$ be an ordinal for $B$, so that

$$
(g \circ f)^{<\alpha} \circ a=g^{<\alpha} \circ f^{<\alpha} \circ a=g^{<\alpha} \circ b \circ f=c \circ g \circ f .
$$

Definition 3.4 (categories of monographs). Let MonoGr be the category of monographs and their morphisms. Let SMonoGr be its full subcategory of standard monographs. For any set $O$ of ordinals, let $O-\mathbf{M o n o G r}$ (resp. O-SMonoGr) be the full subcategory of O-monographs (resp. standard $O$-monographs).

A monograph $A$ is finite if $\mathrm{E}_{a}$ is finite. Let $\mathbf{F M o n o G r}$ be the full subcategory of finite $\omega$-monographs.

It is customary in Algebraic Graph Transformation to call typed graphs the objects of Graphs $/ G$, where $G$ is a graph called type graph, see e.g. 4]. A type graph is therefore seen as the specification of a category of typed graphs. In particular, the terminal graph $G_{t}$ specifies the whole category of graphs since Graphs $\simeq$ Graphs $/ G_{t}$. We will extend this terminology to monographs and refer to the objects of MonoGr/T as the monographs typed by $T$. The monograph $T$ is then considered as a type monograph and hence as a specification for a category of typed monographs.

It is obvious from the above that for any set $O$ of ordinals, if $T$ is an $O$ monograph then MonoGr/T $=O$-MonoGr/T. Similarly, if $f: A \rightarrow B$ then

$$
f^{-1}\left[\mathrm{~N}_{b}\right]=\left\{x \in \mathrm{E}_{a} \mid \ell_{b}(f(x))=0\right\}=\mathrm{N}_{a},
$$

hence if $b \circ f(x)$ is a $\mathrm{N}_{b}$-sequence for some $x \in \mathrm{E}_{a}$ then for all $\iota<\ell_{a}(x)$,

$$
f\left(a(x)_{\iota}\right)=(f \circ a(x))_{\iota}=\left(f^{<\alpha}(a(x))\right)_{\iota}=\left(f^{<\alpha} \circ a(x)\right)_{\iota}=(b \circ f(x))_{\iota} \in \mathrm{N}_{b},
$$

\footnotetext{
${ }^{3}$ Imposing the grade of $A$ for $\alpha$ here would be a useless constraint. Note that the equation $f^{<\alpha} \circ a=b \circ f$ holds for all ordinals $\alpha$ for $A$ iff it holds for one.
} 
hence $a(x)_{\iota} \in \mathrm{N}_{a}$ and $a(x)$ is therefore a $\mathrm{N}_{a}$-sequence. Thus $A$ is standard whenever $B$ is standard. This proves that for any standard monograph (resp. standard $O$-monograph) $T$ we have MonoGr $/ T=\mathbf{S M o n o G r} / T$ (resp. MonoGr $/ T=$ $O$-SMonoGr $/ T)$.

The introduction of the present section suggests a similitude between graphs and standard $\{0,2\}$-monographs. It is actually easy to define a functor $\mathrm{M}$ : Graphs $\rightarrow\{0,2\}$-SMonoGr by mapping any graph $G=(N, E, s, t)$ to $\mathrm{M} G=$ $(N+E, g)$ where $g(x)=\varepsilon$ for all $x \in N$ and $g(e)=s(e) t(e)$ for all $e \in E$ (and similarly graph morphisms are transformed into morphisms of monographs through a coproduct of functions). It is easy to see that $\mathrm{M}$ is an equivalence of categories ${ }^{4}$. This means that for any graph $G$ we have Graphs $/ G \approx$ MonoGr/MG, hence that typed graphs can be represented as typed monographs.

\section{Drawing Monographs}

Obviously we may endeavour to draw a monograph $A$ only if $\mathrm{E}_{a}$ is finite and if its edges have finite lengths, i.e., if $A$ is a finite $\omega$-monograph. If we require that any monograph $\mathrm{M} G$ should be drawn as the graph $G$, then a node should be represented by a bullet $\bullet$ and an edge of length 2 by an arrow $\longrightarrow$ joining its two adjacent nodes. But generally the adjacent edges may not be nodes and there might be more than 2 of them, hence we adopt the following convention: an edge $e$ of length at least 2 is represented as a sequence of connected arrows with an increasing number of tips

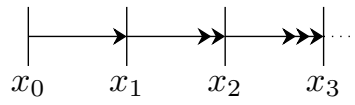

(where $a(e)=x_{0} x_{1} x_{2} x_{3} \cdots$ ) and such that any arrow should enter $x_{i}$ at the same angle as the next arrow leaves $x_{i}$. This is important when $x_{i}$ is a node since several adjacent edges may traverse the corresponding bullet, and they should not be confused. For the sake of clarity we will also represent symmetric adjacencies by a pair of crossings rather than a single one, e.g., if $a(e)=x e^{\prime} y$ and $a\left(e^{\prime}\right)=x e y$, where $x$ and $y$ are nodes, the drawing may be

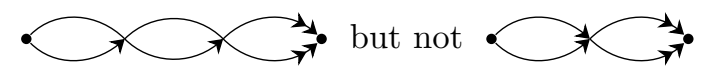

It is sometimes necessary to name the edges in a drawing. We may then adopt the convention used for drawing diagrams in a category: the bullets are replaced by the names of the corresponding nodes, and arrows are interrupted to write their name at a place free from intersection, as in

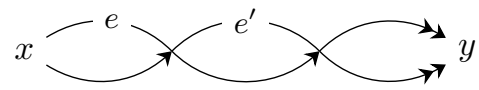

Note that no confusion is possible between the names of nodes and those of other edges, e.g., in

${ }^{4}$ This can be seen as a consequence of Corollary 6.8 below and of the isomorphism between Graphs and the category of $\Sigma_{\mathrm{g}}$-algebras. 


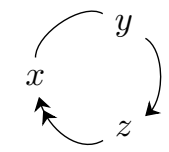

it is clear that $x$ and $z$ are nodes since arrow tips point to them, and that $y$ is the name of an edge of length 3 .

As is the case of graphs, monographs may not be planar and drawing them may require crossing edges that are not adjacent; in this case no arrow tip is present at the intersection and no confusion is possible with the adjacency crossings. However, it may seem preferable in such cases to erase one arrow in the proximity of the other, as in $入$.

There remains to represent the edges of length 1 . Since $a(e)=x$ is standardly written $a: e \mapsto x$, the edge $e$ will be drawn as

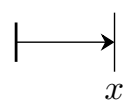

In order to avoid confusion there should be only one arrow out of the thick dash, e.g., if $a(e)=e^{\prime}$ and $a\left(e^{\prime}\right)=e x$ where $x$ is a node, the drawing may be

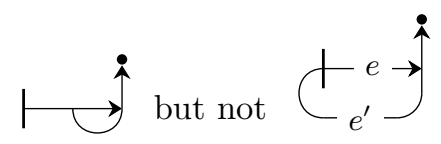

since this last drawing may be interpreted as the monograph $a\left(e^{\prime}\right)=x$ and $a(e)=e^{\prime} e^{\prime}$, that is not isomorphic to the intended monograph.

With these conventions it is only possible to read a drawing of any finite $\omega$-monograph $A$ as the monograph $A$ itself if all edges are named in the drawing, or as some monograph isomorphic to $A$ otherwise. Note that this would not be true if the map $a$ was a function rather than a functional relation, because its codomain $\mathrm{E}_{a}^{<\alpha}$ is not pictured. It would of course be possible to add the ordinal $\alpha$ to the drawing, but then would it still qualify as a drawing?

Note that the drawing of a graph or of a standard $\{0,2\}$-monograph can be read either as a graph $G$ or as a monograph $A$, and then $\mathrm{M} G \simeq A$.

Possible drawings for the self-loops of length 1 to 4 are given below.

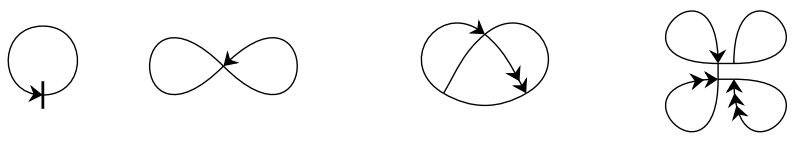

We may also draw typed monographs, i.e., monographs $A$ equipped with a morphism $f$ from $A$ to a monograph $T$, considered as a type. Then every edge $e \in \mathrm{E}_{a}$ has a type $f(e)$ that can be written at the proximity of $e$. For instance, let $T$ be the monograph

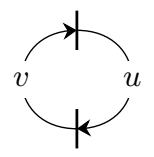


then a monograph typed by $T$ is drawn with labels $u$ and $v$ as in

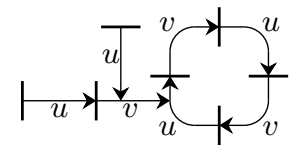

Of course, knowing that $f$ is a morphism sometimes allows to deduce the type of an edge, possibly from the types of adjacent edges. In the present case, indicating a single type would have been enough to deduce all the others.

\section{Monadic Signatures as Monographs}

As mentioned in Section 1, graph structures, i.e., monadic many-sorted signatures, can be represented as graphs. More precisely, there is an obvious isomorphism between the category Graphs and the category of monadic signatures defined below

Definition 5.1 (monadic signatures). $A$ (monadic) signature is a function $\Sigma: \Omega \rightarrow S \times S$; the elements of its domain $\Omega$, that may be written $\Sigma_{\mathrm{op}}$, are called operator names and the elements of $S$, that may be written $\Sigma_{\mathrm{srt}}$, are called sorts. $\Sigma$ is finite if both $\Omega$ and $S$ are finite. Let $\Sigma_{\mathrm{ds}} \stackrel{\text { def }}{=} \pi_{1} \circ \Sigma$ and $\Sigma_{\mathrm{rs}} \stackrel{\text { def }}{=} \pi_{2} \circ \Sigma$, then $\Sigma_{\mathrm{ds}}(o)$ and $\Sigma_{\mathrm{rs}}(o)$ are respectively the domain and range sorts of $o \in \Omega$.

$A$ morphism $m$ from signature $\Sigma$ to signature $\Sigma^{\prime}$ is a pair $m=\left(m_{\mathrm{op}}, m_{\mathrm{srt}}\right)$ of functions, where $m_{\mathrm{op}}: \Sigma_{\mathrm{op}} \rightarrow \Sigma_{\mathrm{op}}^{\prime}$ and $m_{\mathrm{srt}}: \Sigma_{\mathrm{srt}} \rightarrow \Sigma_{\mathrm{srt}}^{\prime}$, such that

$$
\Sigma^{\prime} \circ m_{\mathrm{op}}=\left(m_{\mathrm{srt}} \times m_{\mathrm{srt}}\right) \circ \Sigma \text {. }
$$

Let $\operatorname{id}_{\Sigma} \stackrel{\text { def }}{=}\left(\operatorname{Id}_{\Sigma_{\mathrm{op}}}, \operatorname{Id}_{\Sigma_{\text {srt }}}\right)$ and given two morphisms $m: \Sigma \rightarrow \Sigma^{\prime}$ and $n: \Sigma^{\prime} \rightarrow$ $\Sigma^{\prime \prime}$, let $n \circ m \stackrel{\text { def }}{=}\left(n_{\mathrm{op}} \circ m_{\mathrm{op}}, n_{\mathrm{srt}} \circ m_{\mathrm{srt}}\right)$; then $\operatorname{id}_{\Sigma}: \Sigma \rightarrow \Sigma$ and $n \circ m: \Sigma \rightarrow \Sigma^{\prime \prime}$ are morphisms. Let MonSig be the category of monadic signatures and their morphisms.

The obvious isomorphism from MonSig to Graphs maps every monadic signature $\Sigma: \Omega \rightarrow S \times S$ to the graph $\left(S, \Omega, \Sigma_{\mathrm{ds}}, \Sigma_{\mathrm{rs}}\right)$. But we have seen in Section 1 on E-graphs that this representation of the monadic signature $\Sigma_{\mathrm{e}}$ bears no relation with the expected graphical representations of E-graphs. It would be more natural to represent $\Sigma_{\mathrm{e}}$ as an E-graph, or more precisely as a monograph whose drawing corresponds to that of an E-graph.

Since the image $\Sigma(\Omega)$ is a subset of $S \times S$, it can be viewed as a binary relation on $S$, hence there exists a monograph with $S$ as set of edges whose adjacency relation is exactly $\Sigma(\Omega)$. However, this monograph may not be unique since, as mentioned in Section 3 a monograph is not generally determined by its adjacency relation. Similarly, the direction of edges in E-graphs is not determined by the signature $\Sigma_{\mathrm{e}}$, it is only a convention given by the names of its operators.

\footnotetext{
${ }^{5}$ For the sake of simplicity, we do not allow the overloading of operator names as in 10, which would be irrelevant anyway since we wish to abstract the syntax away, hence to consider signatures only up to isomorphisms.
} 
For this reason it is more convenient to define a function from monographs to monadic signatures. Indeed, any monograph determines a unique adjacency relation that can then be interpreted as a signature. The sorts of this signature are exactly the edges of the monograph, and for every edge

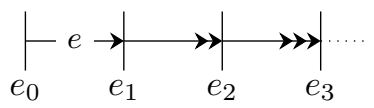

we create a first operator name from sort $e$ to sort $e_{0}$, a second operator name from $e$ to $e_{1}$, and so on for all elements of the sequence.

Definition 5.2 (functor S : MonoGr $\rightarrow$ MonSig). To every monograph $T=$ $\left(\mathrm{E}_{t}, t\right)$ we associate the set

$$
\Omega_{t} \stackrel{\text { def }}{=}\left\{(e, \iota) \mid e \in \mathrm{E}_{t} \wedge \iota<\ell_{t}(e)\right\}
$$

of operator names, and the signature $\mathrm{ST}: \Omega_{t} \rightarrow \mathrm{E}_{t} \times \mathrm{E}_{t}$ defined by

$$
\mathrm{ST}(e, \iota) \stackrel{\text { def }}{=}\left(e, t(e)_{\iota}\right) \text { for all }(e, \iota) \in \Omega_{t} \text {. }
$$

To every morphism of monographs $f: T \rightarrow U$ we associate the morphism $\mathrm{S} f$ : $\mathrm{ST} \rightarrow \mathrm{SU}$ defined by

$-(\mathrm{S} f)_{\mathrm{op}}(e, \iota) \stackrel{\text { def }}{=}(f(e), \iota) \in \Omega_{u}$ for all $(e, \iota) \in \Omega_{t}$, and

- $(\mathrm{S} f)_{\mathrm{srt}} \stackrel{\text { def }}{=} f$ (as a function from $\mathrm{E}_{t}$ to $\mathrm{E}_{u}$ ).

Note that the signature ST is finite iff $T$ is a finite $\omega$-monograph.

Lemma 5.3. S is an embedding from MonoGr to MonSig.

The next lemma uses the Axiom of Choice through its equivalent formulation known as the Numeration Theorem [1].

Lemma 5.4. S is isomorphism-dense: for every monadic signature $\Sigma$ there exists a monograph $T$ such that $\mathrm{ST} \simeq \Sigma$.

Proof (sketch). For any object $\Sigma: \Omega \rightarrow S \times S$ of MonSig, and for every sort $s \in S$ let $O_{s}$ be the set of operator names in $\Omega$ whose domain sort is $s$. By the Numeration Theorem there exists an ordinal $\lambda_{s}$ equipollent to $O_{s}$, i.e., such that there exists a bijection $\sigma_{s}: \lambda_{s} \rightarrow O_{s}$. Let $t(s)$ be the $S$-sequence of length $\lambda_{s}$ such that, for all $\iota<\lambda_{s}, t(s)_{\iota}$ is the range sort of the operator name $\sigma_{s}(\iota)$, and let $T$ be the monograph $(S, t)$. It is then easy to see that the signature ST is isomorphic to $\Sigma$ by mapping every operator name $(s, \iota) \in \Omega_{t}$ to the original operator name $\sigma_{s}(\iota) \in \Omega$.

The reason why monographs require edges of ordinal length now becomes apparent: the length of an edge $s$ is the cardinality of $O_{s}$, i.e., the number of operators whose domain sort is $s$, and no restriction on this cardinality is ascribed to signatures. The bijections $\sigma_{s}$ provide linear orderings of the sets $O_{s}$.

We now show on an example that the functor $S$ is not full, hence that $S$ is not an equivalence between the categories MonoGr and MonSig. 
Example 5.5. The monadic signature $\Sigma_{\mathrm{g}}$ has two operators src, tgt, two sorts in $\mathrm{S}_{\mathrm{g}}=$ \{nodes, edges $\}$ and is defined by $\Sigma_{\mathrm{g}}: \mathrm{src}, \operatorname{tgt} \mapsto$ (edges, nodes). Then $O_{\text {nodes }}=\varnothing$ and $O_{\text {edges }}=\{$ src, tgt $\}$ has 2 elements. Let $\sigma: 2 \rightarrow O_{\text {edges }}$ be the bijection defined by $\sigma: 0 \mapsto \operatorname{src}, 1 \mapsto$ tgt and $t$ be the map defined by

$$
t(\text { nodes })=\varepsilon, t(\text { edges })=\text { nodes nodes }
$$

then $\mathrm{T}_{\mathrm{g}}=\left(\mathrm{S}_{\mathrm{g}}, t\right)$ is a monograph. The signature $\mathrm{ST}_{\mathrm{g}}$ has the same sorts as $\Sigma_{\mathrm{g}}$, two operators (edges, 0 ), (edges, 1 ) and is defined by

$$
\mathrm{ST}_{\mathrm{g}}:(\text { edges, } 0),(\text { edges, } 1) \mapsto(\text { edges, nodes })
$$

Hence $\mathrm{ST}_{\mathrm{g}}$ is indeed isomorphic to $\Sigma_{\mathrm{g}}$. However, the only automorphism of $\mathrm{T}_{\mathrm{g}}$ is $\operatorname{id}_{T_{\mathrm{g}}}$, while $\Sigma_{\mathrm{g}}$ has a non trivial automorphism $m=\left((\operatorname{srctgt}), \operatorname{Id}_{\mathrm{S}_{\mathrm{g}}}\right)$ (in cycle notation), hence $S$ is not surjective on morphisms.

This automorphism reflects the fact that $\Sigma_{\mathrm{g}}$ does not define an order between its operators src and tgt. Directing edges as arrows from src to tgt is only a matter of convention that is reflected in the choice of $\sigma$ above. This contrasts with monographs, where the edges are inherently directed by the ordinals in their length. In the translation from MonoGr to MonSig, the direction of edges are necessarily lost. Note however that in this example, since src and tgt have the same range sort, the other obvious choice for $\sigma$ yields the same monograph $\mathrm{T}_{\mathrm{g}}$.

We therefore see that in most cases there are many distinct, non isomorphic monographs that faithfully represent a single signature, depending on the chosen direction of their edges. Monographs carry more information than signatures, but the additional information is precisely the kind of information that has to be provided by means of syntax when a monadic signature is intended as a graph structure. By observing the examples given in [7, Section 3.1], we see that this syntactic information mostly consists of an order on operators, given either by indices or by calling them "source" and "target".

More precisely, Examples 3.1 to 3.4 of [7] are monadic signatures defining graphs, edge-labelled and labelled graphs (with a possibly infinite set of labels) and hypergraphs (see Example 6.10 below). Examples 3.5 and 3.6 are special representations of signatures and terms. We observe in these examples a strict partition of sorts into domain and range sorts. It is easy to see that a monograph $T$ is standard iff the signature ST is separated, i.e., no sort occurs both as a domain and a range sort. Thus the range sorts are the nodes of $T$ and the domain sorts are edges of diverse lengths that relate nodes.

We now consider in detail Example 3.7 of [7, the only example of a monadic signature that is not separated.

Example 5.6. Let $\Sigma_{\mathrm{a}}$ be the monadic signature defined by the set of sorts

$$
\mathrm{S}_{\mathrm{a}}=\{\mathrm{V}, \mathrm{E}, \mathrm{V}-\text { Ass, E-Ass, Graph, Morphism }\}
$$


and the following operators:

$$
\begin{aligned}
\Sigma_{\mathrm{a}}: \quad \mathrm{s}, \mathrm{t} & \mapsto(\mathrm{E}, \mathrm{V}) \\
\mathrm{s}_{\mathrm{V}}, \mathrm{t}_{\mathrm{V}} & \mapsto(\mathrm{V}-\text { Ass }, \mathrm{V}) \\
\mathrm{s}_{\mathrm{E}}, \mathrm{t}_{\mathrm{E}} & \mapsto(\mathrm{E}-\text { Ass }, \mathrm{E}) \\
\mathrm{s}_{\mathrm{G}}, \mathrm{t}_{\mathrm{G}} & \mapsto(\text { Morphism, Graph) } \\
\text { abstract }_{\mathrm{V}} & \mapsto(\mathrm{V}, \text { Graph }) \\
\text { abstract }_{\mathrm{E}} & \mapsto(\mathrm{E}, \text { Graph }) \\
\text { abstract }_{\mathrm{V}-\mathrm{Ass}} & \mapsto(\mathrm{V}-\text { Ass }, \text { Morphism }) \\
\text { abstract }_{\mathrm{E}-\text { Ass }} & \mapsto(\mathrm{E}-\text { Ass }, \text { Morphism })
\end{aligned}
$$

An ALR-graph is a $\Sigma_{\mathrm{a}}$-algebra. It is not very clear how such structures can be considered as graphs, especially because there is no conventional way of ordering the operator name abstract w.r.t. $s$ and $t$ (we only know that sources come before targets). Textual explanations are provided in [7] to help the reader's understanding of ALR-graphs:

ALR-graphs not only allow to represent arbitrary labeled graphs but also morphisms between graphs. Since morphisms map vertices to vertices and edges to edges, they are represented by pairs of vertex assignments and edge assignments. In order to keep track of which assignment belongs to which morphism, an abstraction operator is introduced in ALR-graphs which allows to group vertices and edges into graphs and vertex and edge assignments into morphisms. Thus, ALR-graphs as algebras w.r.t. the graph structure below are able to represent the diagram level (graphs and morphisms) and the object level (vertices, edges, and assignments) in a single structure.

The explanations given below on the corresponding monograph are much simpler. A choice of ordering abstract between $s$ and $t$ is made, that helps visualize the structure. The set of edges is of course $S_{a}$, and the map $t_{a}$ is defined by:

$$
\begin{array}{ll}
\mathrm{t}_{\mathrm{a}}(\text { Graph })=\varepsilon & \text { graphs are represented by nodes } \\
\mathrm{t}_{\mathrm{a}}(\mathrm{V})=\mathrm{Graph} & \text { to every vertex is associated a graph } \\
\mathrm{t}_{\mathrm{a}}(\mathrm{E})=\mathrm{V} \text { Graph } \mathrm{V} & \text { an edge joins two vertices through a graph } \\
\mathrm{t}_{\mathrm{a}}(\text { Morphism })=\text { Graph Graph } & \text { a morphism joins two graphs } \\
\mathrm{t}_{\mathrm{a}}(\mathrm{V}-\mathrm{Ass})=\mathrm{V} \text { Morphism V } & \text { a vertex association joins two vertices } \\
& \text { through a morphism } \\
\mathrm{t}_{\mathrm{a}}(\mathrm{E}-\text { Ass })=\text { EMorphism } \mathrm{E} & \text { an edge association joins two edges } \\
& \text { through a morphism. }
\end{array}
$$

We thus see that specifying a monadic signature by a monograph may yield a better understanding of the structure of the corresponding algebras, at least if these are meant as graph structures. The next section shows that this is always possible. 


\section{Monadic Algebras as Typed Monographs}

Now that monographs have been embedded in graph structures, the relation that the corresponding algebras bear with these monographs may be investigated. We first need a definition of $\Sigma$-algebras and $\Sigma$-homomorphisms that, for the sake of simplicity, are restricted to monadic signatures.

Definition 6.1 ( $\Sigma$-algebras). For any monadic signature $\Sigma: \Omega \rightarrow S \times S$, a $\Sigma$-algebra $\mathcal{A}$ is a pair $\left(\left(\mathcal{A}_{s}\right)_{s \in S},\left(o^{\mathcal{A}}\right)_{o \in \Omega}\right)$ where $\left(\mathcal{A}_{s}\right)_{s \in S}$ is an $S$-indexed family of sets and $o^{\mathcal{A}}: \mathcal{A}_{\Sigma_{\mathrm{ds}}(o)} \rightarrow \mathcal{A}_{\Sigma_{\mathrm{rs}}(o)}$ is a function for all $o \in \Omega$. $\mathcal{A}$ is partitioned if $s \neq s^{\prime}$ entails $\mathcal{A}_{s} \cap \mathcal{A}_{s^{\prime}}=\varnothing$ for all $s, s^{\prime} \in S$.

$A \Sigma$-homomorphism $h: \mathcal{A} \rightarrow \mathcal{B}$ from a $\Sigma$-algebra $\mathcal{A}$ to a $\Sigma$-algebra $\mathcal{B}$ is an $S$-indexed family of functions $\left(h_{s}\right)_{s \in S}$ where $h_{s}: \mathcal{A}_{s} \rightarrow \mathcal{B}_{s}$ for all $s \in S$, such that

$$
o^{\mathcal{B}} \circ h_{\Sigma_{\mathrm{ds}}(o)}=h_{\Sigma_{\mathrm{rs}}(o)} \circ o^{\mathcal{A}}
$$

for all $o \in \Omega$. Let $\operatorname{id}_{\mathcal{A}}: \mathcal{A} \rightarrow \mathcal{A}$ be the $\Sigma$-homomorphism $\left(\operatorname{Id}_{\mathcal{A}_{s}}\right)_{s \in S}$, and for any $\Sigma$-homomorphism $h: \mathcal{A} \rightarrow \mathcal{B}$ and $k: \mathcal{B} \rightarrow \mathcal{C}$, let $k \circ h: \mathcal{A} \rightarrow \mathcal{C}$ be the $\Sigma$-homomorphism $\left(k_{s} \circ h_{s}\right)_{s \in S}$. Let $\Sigma$-Alg be the category of $\Sigma$-algebras with $\Sigma$-homomorphisms as their morphisms, and $\Sigma$-PAlg be its full subcategory of partitioned algebras.

Following Example 5.5, we notice that $T_{g}=G_{\mathrm{e}} \simeq \mathrm{MG}_{\mathrm{t}}$ (where $\mathrm{G}_{\mathrm{t}}$ is the terminal graph, see Section 3, hence we have

$$
\text { Graphs } \simeq \text { Graphs } / \mathrm{G}_{\mathrm{t}} \approx \mathrm{MonoGr} / \mathrm{MG}_{\mathrm{t}} \simeq \mathrm{MonoGr} / \mathrm{T}_{\mathrm{g}}
$$

But we know that Graphs $\simeq \Sigma_{\mathrm{g}}$-Alg and that $\Sigma_{\mathrm{g}} \simeq \mathrm{ST}$. The following result allows us to replace $\Sigma_{\mathrm{g}}$ by $\mathrm{ST}_{\mathrm{g}}$.

Lemma 6.2. If $\Sigma \simeq \Sigma^{\prime}$ then $\Sigma$-Alg $\simeq \Sigma^{\prime}$-Alg and $\Sigma$-PAlg $\simeq \Sigma^{\prime}$-PAlg.

Note that $\Sigma$-Alg is not isomorphic to $\Sigma$-PAlg since many distinct algebras may be $\Sigma$-isomorphic to the same partitioned algebra. There is however a trivial equivalence between these categories.

Lemma 6.3. For every signature $\Sigma, \Sigma$-PAlg $\approx \Sigma$-Alg

By Lemma 6.2 we thus obtain MonoGr/ $\mathrm{T}_{\mathrm{g}} \approx \mathrm{ST} \mathrm{T}_{\mathrm{g}}$-Alg, the monographs typed by $\mathrm{T}_{\mathrm{g}}$ are essentially the $\mathrm{ST}_{\mathrm{g}}$-algebras. We are now going to generalize this property to all monographs $T$ with the following functor from the category of monographs typed by $T$ to the category of partitioned $S T$-algebras.

Definition 6.4 (functor $\mathrm{A}_{T}:$ MonoGr $/ T \rightarrow$ ST-PAlg). Given a monograph $T$, we define the function $\mathrm{A}_{T}$ that maps every object $f: A \rightarrow T$ of MonoGr/T to the partitioned $\mathrm{ST}$-algebra $\mathrm{A}_{T} f$ defined by

$-\left(\mathrm{A}_{T} f\right)_{e} \stackrel{\text { def }}{=} f^{-1}[e]$ for all $e \in \mathrm{E}_{t}$, and

$-(e, \iota)^{\mathrm{A}_{T} f}(x) \stackrel{\text { def }}{=} a(x)_{\iota}$ for all $x \in f^{-1}[e]$ and $(e, \iota) \in \Omega_{t}$. 


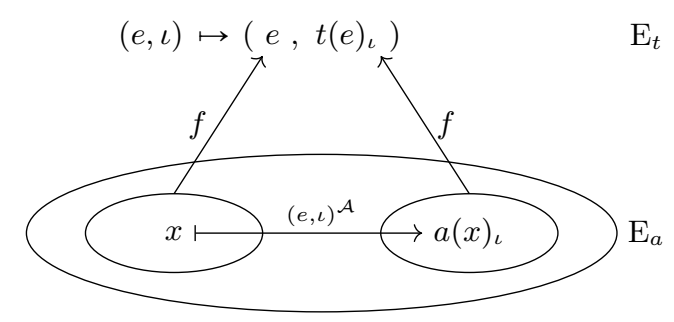

Fig. 1. The ST-algebra $\mathcal{A}=\mathrm{A}_{T} f$ where $f: A \rightarrow T$

Besides, $\mathrm{A}_{T}$ also maps every morphism $k: f \rightarrow g$ of MonoGr/T, where $f$ : $A \rightarrow T$ and $g: B \rightarrow T$, to the $\mathrm{ST}$-homomorphism $\mathrm{A}_{T} k$ from $\mathrm{A}_{T} f$ to $\mathrm{A}_{T} g$ defined by

$$
\left.\left(\mathrm{A}_{T} k\right)_{e} \stackrel{\text { def }}{=} k\right|_{f^{-1}[e]} \text { for all } e \in \mathrm{E}_{t} .
$$

The ST-algebra $\mathcal{A}=\mathrm{A}_{T} f$ can be pictured as in Figure 1. In ST, every operator name $(e, \iota)$ has $e$ and $t(e)_{\iota}$ as domain and range sort respectively, and these two sorts are also edges in $T$. Hence these edges have inverse images by $f$ in $\mathrm{E}_{a}$ (they form a partion of the set $\mathrm{E}_{a}$ ). The inverse image of an edge $e$ of $T$ is considered as the carrier set $\mathcal{A}_{e}$, since $e$ is a sort of ST. The operator name $(e, \iota)$ is interpreted as the function $(e, \iota)^{\mathcal{A}}$ that maps every $x \in f^{-1}[e]$ to the edge $a(x)_{\iota}$.

Example 6.5. Consider the monograph $\mathrm{T}_{\mathrm{g}}$, the corresponding signature $\mathrm{ST}_{\mathrm{g}}$ from Example 5.5, and the monograph $A=(\{x, y, z\}, a)$ where $a(x)=y z$ and $a(y)=a(z)=\varepsilon$, i.e., $x$ is an edge from node $y$ to node $z$. There is a unique morphism $f: A \rightarrow \mathrm{T}_{\mathrm{g}}$, that is $f(x)=$ edges and $f(y)=f(z)=$ nodes.

Let $\mathcal{A}$ be the $\mathrm{ST}_{\mathrm{g}}$-algebra $\mathrm{A}_{\mathrm{T}_{\mathrm{g}}} f$. By Definition 6.4 we have

$$
\mathcal{A}_{\text {edges }}=f^{-1}[\text { edges }]=\{x\} \text { and } \mathcal{A}_{\text {nodes }}=f^{-1}[\text { nodes }]=\{y, z\} .
$$

$\mathrm{ST}_{\mathrm{g}}$ has two operator names (edges,0) and (edges, 1 ), both of domain sort edges and range sort nodes, and their interpretation in $\mathcal{A}$ is given by

$$
(\text { edges }, 0)^{\mathcal{A}}(x)=a(x)_{0}=y \text { and }(\text { edges }, 1)^{\mathcal{A}}(x)=a(x)_{1}=z .
$$

Thus $\mathcal{A}$ is the algebra that traditionally represents $A$ considered as a graph (more precisely, as a graph $G$ such that $\mathrm{M} G \simeq A$ ).

But the important point is that not only the monograph $A$ but also its typing $f: A \rightarrow T$ can be uniquely reconstructed from any partitioned ST-algebra $\mathcal{A}$, and that this is also true on morphisms. In other words, $A_{T}$ is not just a functor, it is an isomorphism of categories.

Theorem 6.6. For every monograph $T, \mathrm{~A}_{T}:$ MonoGr $/ T \stackrel{\simeq}{\rightarrow} \mathrm{S} T$-PAlg.

Corollary 6.7. For every monadic signature $\Sigma$ there exists a monograph $T$ such that $\Sigma$-PAlg $\simeq$ MonoGr $/ T$. 
Proof. By Lemma 5.4 there exists $T$ such that $\Sigma \simeq \mathrm{ST}$, hence MonoGr/T $\simeq$ ST-PAlg $\simeq \Sigma$-PAlg by Lemma 6.2 .

We thus see that the categories of partitioned monadic algebras are isomorphic to the slice categories of monographs. Note that in the case of graphs, the partitioned $\Sigma_{\mathrm{g}}$-algebras correspond to those graphs whose sets of vertices and edges are disjoint. This is a common restriction for graphs but not for $\Sigma$-algebras. By Lemma 6.3 we obtain a similar result for the categories $\Sigma$-Alg.

Corollary 6.8. For every monograph $T$, MonoGr/T $\cong \mathrm{S} T$-Alg, and for every monadic signature $\Sigma$ there is a monograph $T$ such that $\Sigma$-Alg $\approx$ MonoGr $/ T$.

The first half of Corollary 6.8 also yields that Graphs $/ G \cong \mathrm{S} \circ \mathrm{M}(G)-\mathrm{Alg}$ for all graphs $G$, i.e., typed graphs are equivalent to algebras of monadic signatures (but not every monadic signature is isomorphic to some $\mathrm{S} \circ \mathrm{M}(G)$ ). Signatures are sometimes called types (see, e.g., 2, Chapter 9]), which leads to the following reading of Corollary 6.8

algebras of monadic many-sorted types are essentially typed monographs.

This may seem strange since monographs may be typed in many different ways, while algebras are defined with only one type (but this type may correspond to many different type monographs). We now illustrate the corresponding equivalence between E-graphs and a category of typed monographs.

Example 6.9. The signature $\Sigma_{\mathrm{e}}$ of E-graphs from [4 has six operators $\operatorname{src}_{\mathrm{g}}$, $\operatorname{tgt} t_{g}, \operatorname{src}_{n}, \operatorname{tgt} t_{n}, \operatorname{src}_{e}, \operatorname{tgt}_{e}$ and five sorts in

$$
\mathrm{S}_{\mathrm{e}}=\left\{\text { edges }_{\mathrm{g}}, \text { edges }_{\mathrm{n}}, \text { edges }_{\mathrm{e}}, \text { nodes }_{\mathrm{g}}, \text { nodes }_{\mathrm{v}}\right\},
$$

and is defined by

$$
\begin{aligned}
\Sigma_{\mathrm{e}}: \operatorname{src}_{\mathrm{g}}, \operatorname{tgt}_{\mathrm{g}} & \mapsto\left(\text { edges }_{\mathrm{g}}, \text { nodes }_{\mathrm{g}}\right) \\
\operatorname{src}_{\mathrm{n}} & \mapsto\left(\text { edges }_{\mathrm{n}}, \text { nodes }_{\mathrm{g}}\right) \\
\operatorname{tgt}_{\mathrm{n}} & \mapsto\left(\text { edges }_{\mathrm{n}}, \text {,odes }_{\mathrm{v}}\right) \\
\operatorname{src}_{\mathrm{e}} & \mapsto\left(\text { edges }_{\mathrm{e}}, \text { edges }_{\mathrm{g}}\right) \\
\operatorname{tgt}_{\mathrm{e}} & \mapsto\left(\text { edges }_{\mathrm{e}}, \text { nodes }_{\mathrm{v}}\right)
\end{aligned}
$$

hence $O_{\text {edges }_{\mathrm{g}}}=\left\{\mathrm{src}_{\mathrm{g}}, \operatorname{tgt}_{\mathrm{g}}\right\}, O_{\text {edges }_{\mathrm{n}}}=\left\{\mathrm{src}_{\mathrm{n}}, \operatorname{tgt}_{\mathrm{n}}\right\}, O_{\text {edges }_{\mathrm{e}}}=\left\{\mathrm{src}_{\mathrm{e}}, \operatorname{tgt}_{\mathrm{e}}\right\}$ and $O_{\text {nodes }_{\mathrm{g}}}=O_{\text {nodes }_{\mathrm{v}}}=\varnothing$. There are four possible monographs $T=\left(\mathrm{S}_{\mathrm{e}}, t\right)$, given by

$$
\begin{aligned}
& t\left(\text { nodes }_{\mathrm{g}}\right)=t\left(\text { nodes }_{\mathrm{v}}\right)=\varepsilon \\
& t\left(\text { edges }_{\mathrm{g}}\right)=\text { nodes }_{\mathrm{g}} \text { nodes }_{\mathrm{g}} \\
& t\left(\text { edges }_{\mathrm{n}}\right)=\text { nodes }_{\mathrm{g}} \text { nodes }_{\mathrm{v}} \text { or nodes } \\
& t\left(\text { ndges }_{\mathrm{e}}\right)=\text { edges }_{\mathrm{g}} \text { nodes }_{\mathrm{v}} \text { or nodes }_{\mathrm{v}} \text { edges }_{\mathrm{g}} .
\end{aligned}
$$

These four monographs are depicted below.

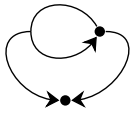

$T_{1}$

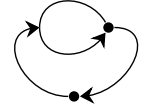

$T_{2}$

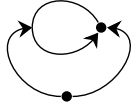

$T_{3}$

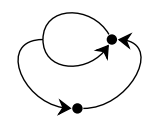

$T_{4}$ 
Note that, by Theorem 6.6 the categories MonoGr/ $/ T_{i}$ for $1 \leqslant i \leqslant 4$ are isomorphic, even though the $T_{i}$ 's are not. The type indicated by the syntax (and consistent with the figures in [4]) is $T_{1}$. An example of a monograph $A$ typed by $T_{1}$ is

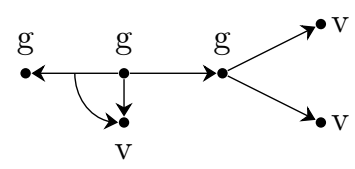

where $\mathrm{g}$ stands for nodes $\mathrm{g}$ and $\mathrm{v}$ for nodes $\mathrm{v}$. The types of the other edges can easily be deduced, yielding a unique typing morphism $f: A \rightarrow T_{1}$. This drawing can also be read as an E-graph, i.e., as a $\Sigma_{\mathrm{e}}$-algebra $\mathcal{E}$ by determining the carrier sets of the five sorts and the functions interpreting the six operator names of $\Sigma_{\mathrm{e}}$. This is a tedious task that corresponds to the transformation performed by the functor $\mathrm{A}_{T_{1}}$, since $\mathcal{E} \simeq \mathrm{A}_{T_{1}} f$ as the reader can check.

Conversely, drawing an E-graph $\mathcal{E}$ corresponds to computing the typed monograph $\mathrm{A}_{T_{1}}^{-1} \mathcal{E}$ and then drawing it. Note that the operator names of $\Sigma_{\mathrm{e}}$ do not appear in the drawing of $\mathcal{E}$ but the sorts do, exactly as in $\mathrm{A}_{T_{1}}^{-1} \mathcal{E}$.

We see that E-graphs can be defined simply by drawing $T_{1}$, just as typed graphs can be defined by drawing their type graph. Of course, it is not always easy to draw a monograph, and it can be more convenient to give it explicitly as in Example 5.6, or indeed in the following example where the type monograph is infinite, though standard and with only one node. This example illustrates how a monograph may be typed in many different ways.

Example 6.10. The signature $\Sigma_{\mathrm{h}}$ of hypergraphs (see [7, Example 3.4]) is defined by the set of sorts $\mathrm{S}_{\mathrm{h}}=\{\mathrm{V}\} \cup\left\{\mathrm{H}_{n, m} \mid n, m \in \omega\right\}$ and the $n+m$ operators

$$
\Sigma_{\mathrm{h}}: \operatorname{src}_{i}^{n, m}, \operatorname{tgt}_{j}^{n, m} \mapsto\left(\mathrm{H}_{n, m}, \mathrm{~V}\right) \text { for all } 1 \leqslant i \leqslant n, 1 \leqslant j \leqslant m .
$$

For any hypergraph $\mathcal{H}$ (i.e., any $\Sigma_{\mathrm{h}}$-algebra) and $n, m \in \omega$, let us call $(n, m)$ hyperedges the elements of the set $\mathcal{H}_{\mathrm{H}_{n, m}}$; these are the hyperedges with $n$ sources and $m$ targets. The corresponding type monograph $\mathrm{T}_{\mathrm{h}}=\left(\mathrm{S}_{\mathrm{h}}, \mathrm{t}_{\mathrm{h}}\right)$ is defined by

$\mathrm{t}_{\mathrm{h}}(\mathrm{V})=\varepsilon \quad$ vertices are nodes

$t_{\mathrm{h}}\left(\mathrm{H}_{n, m}\right)=\mathrm{V}^{n+m}(n, m)$-hyperedges are edges joining $n+m$ vertices for all $n, m \in \omega$. Hypergraphs are therefore isomorphic to monographs typed by $\mathrm{T}_{\mathrm{h}}$, i.e., every edge is typed by some $\mathrm{H}_{n, m}$ (or $\mathrm{V}$ if it is a node). An edge of length 2 can therefore be typed either by $\mathrm{H}_{2,0}, \mathrm{H}_{1,1}$ or $\mathrm{H}_{0,2}$ and thus represent either a $(2,0)$-, a $(1,1)$ - or a $(0,2)$-hyperedge.

\section{Some Properties of MonoGr}

Depending on the signature $\Sigma$, working with $\Sigma$-algebras can be cumbersome. For instance, the proofs given in [4, Chapter 11] have to deal with the five carrier sets and six operators of E-graphs. With only one carrier set and one 
map, monographs allow simpler notations and proofs. This can be illustrated by establishing a few fundamental properties of MonoGr (see e.g. [5] or [1] for the following notions from Category Theory that are not defined in Section 2).

By Corollary 6.8 it would be surprising if MonoGr had a terminal object, since such a monograph would be a type for all monographs, hence the corresponding signature would be in a sense universal. A more direct argument is given below.

Definition 7.1 (monographs $\mathrm{M}_{\alpha}$ ). For every ordinal $\alpha>0$ let $\mathrm{a}_{\alpha}$ be the functional relation that to every $\lambda<\alpha$ associates the unique $\{0\}$-sequence of length $\lambda$. Let $\mathrm{M}_{\alpha} \stackrel{\text { def }}{=}\left(\alpha, \mathrm{a}_{\alpha}\right)$.

It is clear that $\mathrm{M}_{\alpha}$ is a standard $\alpha$-monograph, since $\mathrm{a}_{\alpha} \subseteq \alpha \times \alpha^{<\alpha}$ and $\mathrm{a}_{\alpha}(0)=\varepsilon$, i.e., 0 is a node of $\mathrm{M}_{\alpha}$.

Lemma 7.2. For all ordinals $\alpha>0, \beta$ and every $\beta$-monograph $B$, if there is a morphism $f: \mathrm{M}_{\alpha} \rightarrow B$ then $\alpha \leqslant \beta$.

Proof. $\alpha$ is the grade of $\mathrm{M}_{\alpha}$, since for any $\lambda<\alpha$ there is an edge of length $\lambda$, that is $\ell_{\mathrm{a}_{\alpha}}(\lambda)=\lambda$, hence $\mathrm{a}_{\alpha}(\lambda) \notin \alpha^{<\lambda}$, and therefore $\mathrm{M}_{\alpha}$ is not a $\lambda$-monograph. By the existence of $f$ the grade $\alpha$ of $\mathrm{M}_{\alpha}$ is less than the grade of $B$, hence $\alpha \leqslant \beta$.

Theorem 7.3. MonoGr, SMonoGr and FMonoGr have no terminal object.

Proof. Suppose that $B$ is a terminal monograph, then there is an ordinal $\beta$ such that $B$ is a $\beta$-monograph, and there is a morphism from $\mathrm{M}_{\beta+1}$ to $B$. By Lemma 7.2 this implies that $\beta+1 \leqslant \beta$, a contradiction. This still holds if $B$ is standard since $\mathrm{M}_{\beta+1}$ is standard. And it also holds if $B$ is a finite $\omega$-monograph, since then $\beta$ can be chosen finite, and then $\mathrm{M}_{\beta+1}$ is also a finite $\omega$-monograph.

This of course is a major difference between MonoGr and Graphs, but also with the category Sets (whose terminal object is 1). Other properties derive directly from those of Sets. This can be illustrated by using the standard construction of pullbacks in Sets for building pullbacks of monographs.

Lemma 7.4. Let $B, C, D$ be $\alpha$-monographs and $f: B \rightarrow D, g: C \rightarrow D$ be morphisms, then there exists an $\alpha$-monograph $A$ and morphisms $g^{\prime}: A \rightarrow B$, $f^{\prime}: A \rightarrow C$ such that $\left(A, f^{\prime}, g^{\prime}\right)$ is a pullback of $(f, g, D)$ in MonoGr.

Proof. Let $E=\left\{(y, z) \in \mathrm{E}_{b} \times \mathrm{E}_{c} \mid f(y)=g(z)\right\}, g^{\prime}=\left.\pi_{1}\right|_{E}, f^{\prime}=\left.\pi_{2}\right|_{E}$ and $A=(E, a)$, where $a$ maps every $x \in E$ to $\left\langle b \circ g^{\prime}(x), c \circ f^{\prime}(x)\right\rangle$.

We first prove that $A$ is an $\alpha$-monograph, i.e., that $a(x) \in E^{<\alpha}$ for all $x \in E$. Let $y=g^{\prime}(x)$ and $z=f^{\prime}(x)$ (so that $x=(y, z)$ ), then $\ell_{b}(y)=\ell_{d}(f(y))=$ $\ell_{d}(g(z))=\ell_{c}(z)$, i.e., $b \circ g^{\prime}(x)$ and $c \circ f^{\prime}(x)$ have the same length $\lambda<\alpha$. Then, for all $\iota<\lambda$,

$$
\begin{aligned}
f\left(\left(b \circ g^{\prime}(x)\right)_{\iota}\right)=\left(f^{<\alpha} \circ b(y)\right)_{\iota} & =(d \circ f(y))_{\iota} \\
& =(d \circ g(z))_{\iota}=\left(g^{<\alpha} \circ c(z)\right)_{\iota}=g\left(\left(c \circ f^{\prime}(x)\right)_{\iota}\right),
\end{aligned}
$$


hence $a(x)_{\iota}=\left(\left(b \circ g^{\prime}(x)\right)_{\iota},\left(c \circ f^{\prime}(x)\right)_{\iota}\right) \in E$, so that $a(x) \in E^{\lambda} \subseteq E^{<\alpha}$.

It is obvious that $g^{\prime<\alpha} \circ a(x)=b \circ g^{\prime}(x)$ and $f^{\prime<\alpha} \circ a(x)=c \circ f^{\prime}(x)$, hence $g^{\prime}: A \rightarrow B$ and $f^{\prime}: A \rightarrow C$ are morphisms. There remains to prove that $\left(A, g^{\prime}, f^{\prime}\right)$ is a pullback of $(f, g, D)$.

Let $A^{\prime}$ be a monograph and $g^{\prime \prime}: A^{\prime} \rightarrow B, f^{\prime \prime}: A^{\prime} \rightarrow C$ be morphisms such that $f \circ g^{\prime \prime}=g \circ f^{\prime \prime}$. Since $\left(E, f^{\prime}, g^{\prime}\right)$ is a pullback of $\left(f, g, \mathrm{E}_{d}\right)$ in Sets (see [4]) then there exists a unique function $h$ from $\mathrm{E}_{a^{\prime}}$ to $E$ such that $g^{\prime \prime}=g^{\prime} \circ h$ and $f^{\prime \prime}=f^{\prime} \circ h$. Then, for all $x \in \mathrm{E}_{a^{\prime}}$,

$$
\begin{aligned}
a \circ h(x) & =\left\langle b \circ g^{\prime} \circ h(x), c \circ f^{\prime} \circ h(x)\right\rangle \\
& =\left\langle b \circ g^{\prime \prime}(x), c \circ f^{\prime \prime}(x)\right\rangle \\
& =\left\langle g^{\prime \prime<\alpha} \circ a^{\prime}(x), f^{\prime \prime<\alpha} \circ a^{\prime}(x)\right\rangle \\
& =\left\langle g^{\prime<\alpha} \circ h^{<\alpha} \circ a^{\prime}(x), f^{\prime<\alpha} \circ h^{<\alpha} \circ a^{\prime}(x)\right\rangle \\
& =\left\langle\pi_{1} \circ\left(h^{<\alpha} \circ a^{\prime}(x)\right), \pi_{2} \circ\left(h^{<\alpha} \circ a^{\prime}(x)\right)\right\rangle \\
& =h^{<\alpha} \circ a^{\prime}(x)
\end{aligned}
$$

hence $h: A^{\prime} \rightarrow A$ is a morphism in MonoGr.

Theorem 7.5. The categories MonoGr, SMonoGr, FMonoGr, $O$-MonoGr and $O$-SMonoGr have pullbacks for every set $O$ of ordinals.

Proof. By Lemma 7.4 and since $A$ is finite (resp. standard, resp. an $O$-monograph) if so are $B$ and $C$.

Corollary 7.6. The monomorphisms in MonoGr are the injective morphisms.

Proof. Assume $f: B \rightarrow D$ is a monomorphism and let $C=B, g=f$ and $\left(A, f^{\prime}, g^{\prime}\right)$ be the pullback of $(f, g, D)$ defined in the proof of Lemma 7.4 then $f \circ g^{\prime}=f \circ f^{\prime}$ hence $\left.\pi_{1}\right|_{\mathrm{E}_{a}}=g^{\prime}=f^{\prime}=\left.\pi_{2}\right|_{\mathrm{E}_{a}}$. For all $x, y \in \mathrm{E}_{b}$, if $f(x)=f(y)$ then $(x, y) \in \mathrm{E}_{a}$ and $x=g^{\prime}(x, y)=f^{\prime}(x, y)=y$, hence $f$ is injective. The converse is obvious.

Another way of stating this last property is that the forgetful functor from MonoGr to Sets reflects monomorphisms (and since it is faithful it obviously preserves them as well).

Similarly the standard construction of pushouts in Sets can be used to build pushouts in MonoGr (see [3]). Since $(\varnothing, \varnothing)$ is an initial object in MonoGr (as in all the considered subcategories), we get the following property.

Theorem 7.7. The categories MonoGr, SMonoGr, FMonoGr, $O$-MonoGr and $O$-SMonoGr are finitely co-complete for every set $O$ of ordinals.

Note that the categories mentioned in Theorem 7.3 are not finitely complete since limits of empty diagrams are terminal objects.

As above, the construction of pushouts can be used to establish that the epimorphisms in MonoGr are exactly the surjective morphisms (i.e., the forgetful functor also reflects epimorphisms). But then it is easy to see that the 
isomorphisms in MonoGr are exactly the bijective morphisms: if $f: A \rightarrow B$ and $g: B \rightarrow A$ are such that $g \circ f=\operatorname{id}_{A}$ and $f \circ g=\operatorname{id}_{B}$, then $f$ is bijective since the underlying functions of $\mathrm{id}_{A}$ and $\mathrm{id}_{B}$ are $\operatorname{Id}_{\mathrm{E}_{a}}$ and $\operatorname{Id}_{\mathrm{E}_{b}}$. Hence MonoGr is obviously balanced, i.e., its isomorphisms are exactly the morphisms that are both mono and epimorphisms.

Since the forgetful functor reflects both mono and epimorphisms, this obviously means that it reflects isomorphisms. This has an interesting consequence.

Lemma 7.8. The forgetful functor from MonoGr preserves and reflects pullbacks and pushouts.

Proof. We first see that the forgetful functor preserves pullbacks. Let $(f, g, D)$ be a sink in MonoGr and $\left(A^{\prime}, f^{\prime \prime}, g^{\prime \prime}\right)$ be a pullback of $(f, g, D)$. Let $\left(A, f^{\prime}, g^{\prime}\right)$ be the pullback of $(f, g, D)$ constructed in the proof of Lemma 7.4, then there is an isomorphism $i: A^{\prime} \rightarrow A$ such that $f^{\prime \prime}=f^{\prime} \circ i$ and $g^{\prime \prime}=g^{\prime} \circ i$. Since $\left(\mathrm{E}_{a}, f^{\prime}, g^{\prime}\right)$ is a pullback of $\left(f, g, \mathrm{E}_{d}\right)$ in Sets, then so is $\left(\mathrm{E}_{a^{\prime}}, f^{\prime \prime}, g^{\prime \prime}\right)$.

Since the forgetful functor is faithful, reflects isomorphisms and preserves pullbacks, then [5, Theorem 24.7] it also reflects them. The case for pushouts is similar.

This means that properties of pullbacks and pushouts in Sets can easily be transfered to MonoGr. Of particular importance in the Double Pushout approach to Algebraic Graph Transformation is the notion of adhesive category from [6].

Definition 7.9. A pushout square $(A, B, C, D)$ is a van Kampen square if for any commutative cube

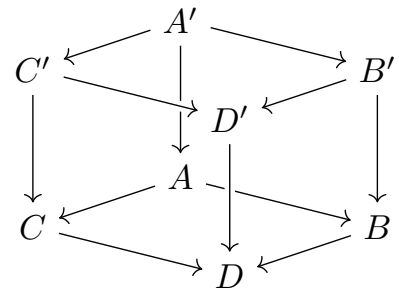

where the back faces $\left(A^{\prime}, A, B^{\prime}, B\right)$ and $\left(A^{\prime}, A, C^{\prime}, C\right)$ are pullbacks, it is the case that the top face $\left(A^{\prime}, B^{\prime}, C^{\prime}, D^{\prime}\right)$ is a pushout iff the front faces $\left(B^{\prime}, B, D^{\prime}, D\right)$ and $\left(C^{\prime}, C, D^{\prime}, D\right)$ are both pullbacks.

A category has pushouts along monomorphisms if all sources $(A, f, g)$ have pushouts whenever $f$ or $g$ is a monomorphism.

A category is adhesive [6] if it has pullbacks, pushouts along monomorphisms and all such pushouts are van Kampen squares.

Theorem 7.10. The categories MonoGr, SMonoGr, FMonoGr, $O$-MonoGr and $O$-SMonoGr are adhesive for every set $O$ of ordinals. 
Proof. In any of these categories a commutative cube built on a pushout along a monomorphism as bottom face and with pullbacks as back faces, has an underlying cube in Sets that has the same properties by Lemma 7.8 and Corollary 7.6 . Since Sets is an adhesive category (see [6]) the underlying bottom face is a van Kampen square, hence such is the bottom face of the initial cube by Lemma 7.8. We conclude with Theorems 7.5 and 7.7 .

Note that their slice categories are therefore also adhesive by [4, Theorem 4.15]. Other properties can be found in [3], in particular a gluing condition that characterizes the existence of a pushout complement, and a construction of a product for some pairs of monographs.

\section{Conclusion}

Monographs generalize standard notions of directed graphs in the following ways.

1. Nodes are considered as a special kind of edges, namely the edges of length zero.

2. Edges can be adjacent to any edges and not only to nodes. This means that a monograph may contain edges but no nodes. This also means that edges can loop on themselves.

3. Edges can have any length, including infinite ones. Similarly (but this is not a special feature) monographs can be infinite.

4. Edges are well-ordered, hence they generalize directed arrows.

The prefix of "monograph" is justified by this unified view of nodes as edges and indiscriminate adjacency that provide formal conciseness; the suffix is justified by the correspondence (up to isomorphism) between finite $\omega$-monographs and their drawings.

Monographs are universal with respect to monadic many sorted signatures (or graph structures) and the corresponding algebras, in the sense that monographs are equivalent to graph structures extended with suitable ordering conventions on their operator names, and that categories of typed monographs are equivalent to the corresponding categories of algebras. Since many standard or exotic notions of directed graphs can be represented as monadic algebras, they can also be represented as typed monographs.

Drawing a typed monograph appears to be a much easier task than drawing an algebra whose signature is a graph structure. This suggests that working directly with typed monographs rather than monadic algebras would be closer to graphical intuition as well as formally simpler.

Future work includes determining further properties of categories of monographs, especially in relation with the Double-Pushout approach to Algebraic Graph Transformation, studying submonographs and partial morphisms [9], attributed monographs as a way of generalizing the notion of attributed graphs based on E-graphs (see [4, Definition 8.4], see also [8]), and their relations with the Single-Pushout approach [7]. 


\section{References}

1. Adámek, J., Herrlich, H., Strecker, G.E.: Abstract and Concrete Categories - The Joy of Cats. Online Edition (2004), http://katmat.math.uni-bremen.de/acc/

2. Bergman, G.M.: An Invitation to General Algebra and Universal Constructions. Springer Universitext (2015), http://dx.doi.org/10.1007/978-3-319-11478-1

3. Boy de la Tour, T.: On monographs, monadic many-sorted algebras and graph structures. HAL (hal-02428793) (2020)

4. Ehrig, H., Ehrig, K., Prange, U., Taentzer, G.: Fundamentals of Algebraic Graph Transformation. Monographs in Theoretical Computer Science. An EATCS Series, Springer (2006)

5. Herrlich, H., Strecker, G.E.: Category Theory. Heldermann Verlag, Berlin, third edn. (2007), http://www.heldermann.de/SSPM/SSPM01/sspm01.htm

6. Lack, S., Sobocinski, P.: Adhesive and quasiadhesive categories. Informatique Théorique et Applications 39(3), 511-545 (2005)

7. Löwe, M.: Algebraic approach to single-pushout graph transformation. Theoretical Computer Science 109, 181-224 (1993)

8. Löwe, M., Korff, M., Wagner, A.: An algebraic framework for the transformation of attributed graphs. In: Sleep, R., Plasmeijer, R., van Eekelen, M. (eds.) Term Graph Rewriting: Theory and Practice, pp. 185-199. John Wiley, New York (1993)

9. Robinson, E., Rosolini, G.: Categories of partial maps. Information and Computation 79(2), 95-130 (Nov 1988)

10. Sannella, D., Tarlecki, A.: Foundations of Algebraic Specification and Formal Software Development. Monographs in Theoretical Computer Science. An EATCS Series, Springer (2012)

11. Suppes, P.: Axiomatic Set Theory. Dover Publications, Inc. (1972) 